\title{
Development of the Floating Centrifugal Pump by Use of Non Contact Magnetic Drive and Its Performance
}

\author{
Mitsuo Uno and Takaaki Masuzoe \\ Kyushu Kyoritsu University, Kitakyushu, Japan
}

Isamu Aotani

M. E. Engi. Lab. Co. Ltd., Kitakyushu, Japan

Shin Oba and Toshiaki Kanemoto

Kyushu Institute of Technology, Kitakyushu, Japan

This article focuses on the impeller construction, non contact driving method and performance of a newly developed shaftless floating pump with centrifugal impeller. The drive principle of the floating impeller pump used the magnet induction method similar to the levitation theory of the linear motor. In order to reduce the axial thrust by the pressure different between shroud and disk side, the balance hole and the aileron blade were installed in the floating impeller. Considering the above effect, floating of an impeller in a pump was realized. Moreover, the performance curves of a developed pump are in agreement with a general centrifugal pump, and the dimensionless characteristic curve also agrees under the different rotational speed due to no mechanical friction of the rotational part. Therefore, utility of a non contacting magnetic-drive style pump with the floating impeller was made clear.

Keywords Centrifugal pump, Drive principle, Floating distance, Magnetic induction, Performance test, Shaftless floating impeller

\section{INTRODUCTION}

For the purpose of maintaining quality and to control the problem of sanitation, the pump for the transportation of functional

Received 25 June 2002; accepted 1 July 2002.

This study was supported by the special research aid of Kyushu Kyoritsu University. The authors appreciate the dedicated assistance in the experimental work by M. S. Uchida, T. Kawai, and K. Taniguchi.

Address correspondence to Professor Mitsuo Uno, Department of Mechanical Engineering, Kyushu Kyoritsu University, Jiyugaoka 1-8, Yahata-nishi, Kitakyushu, 807-8585, Japan. E-mail: mitsuo@ kyulkyo-u.ac.jp materials, food, medical supplies, cleaning solvent, and blood is driven in the sealing condition (Akamatsu, 1994). Hitherto, the magnet drive pump, canned motor pump, and diaphragm pump were used for conveying these fluids. However, a problem may arise in these pumps with the complicated structure in regards to maintenance, durability, and stability. Consequently, in order to prevent contamination, the pump impeller must make the structure, which does not touch on the stationaly part. For the purpose of problem solving, it is newly necessary to develop the floating impeller pump in drive shaftless.

For realization of this pump, the shaftless centrifugal pump with floating impeller was developed to utilize the levitation theory of the linear motor based on the electromagnetism. However, the axial thrust load by pressure difference between shroud and disk side arises in the centrifugal pump impeller (Pfleiderer, 1961; Stepanoff, 1948). Therefore, the axial thrust load must be reduced for the realization of the floating impeller in addition to the holding force of the magnet.

The aim of this article is to develop the shaftless floating impeller pump with the passive control function considering magnetic and hydrodynamic force. Figure 1 shows a developed floating centrifugal pump in order to achieve the above purpose. In this article, the progress of development, pump structure, and magnet induction are explained. Moreover, the possibility of a non contacting magnetic-drive style pump with the floating impeller was examined from observation of operating state and performance test results.

\section{THE DRIVING METHOD OF THE FLOATING IMPELLER}

The drive principle of the floating impeller pump is similar to the levitation theory of the linear motor, as is shown in Figure 2. At the beginning, the rotating magnetic field on the secondary 


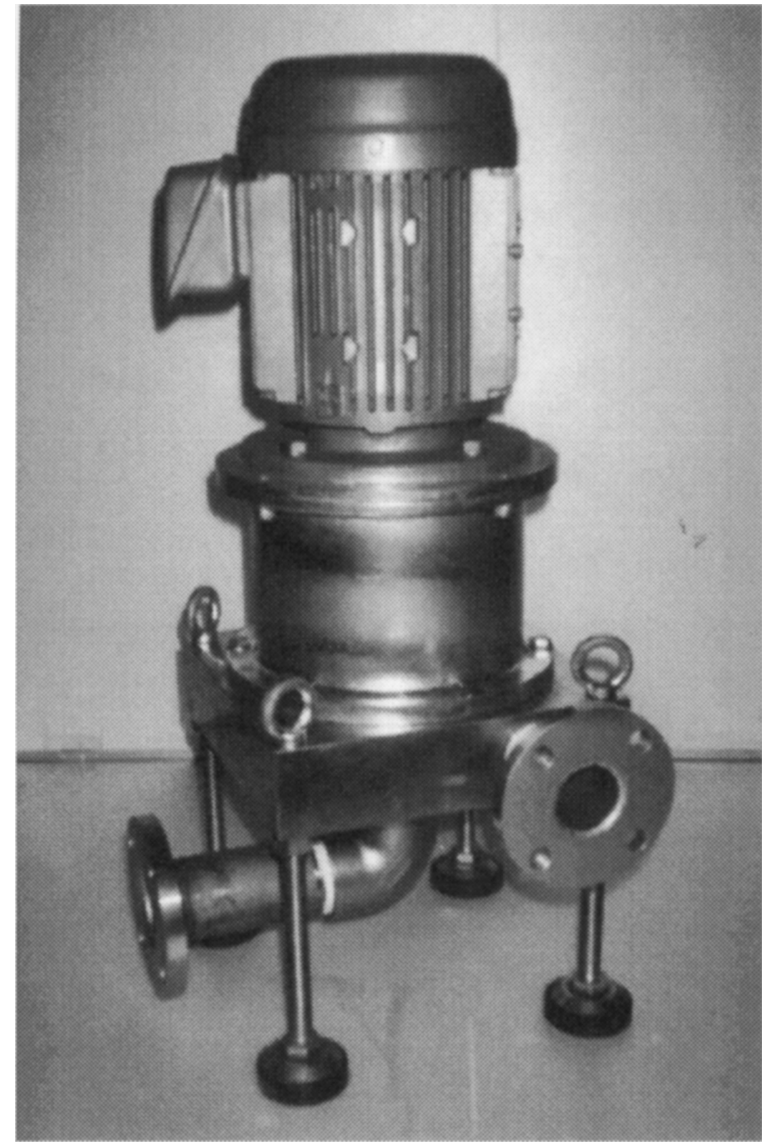

FIGURE 1

Developed floating centrifugal pump.

rotor caused by the eddy current is generated by the rotation of driving magnet. By the phase of both magnetic fields, traction force and normal force occurred on the secondary rotor with impeller. Magnetic flux density of a progressive wave shown in the

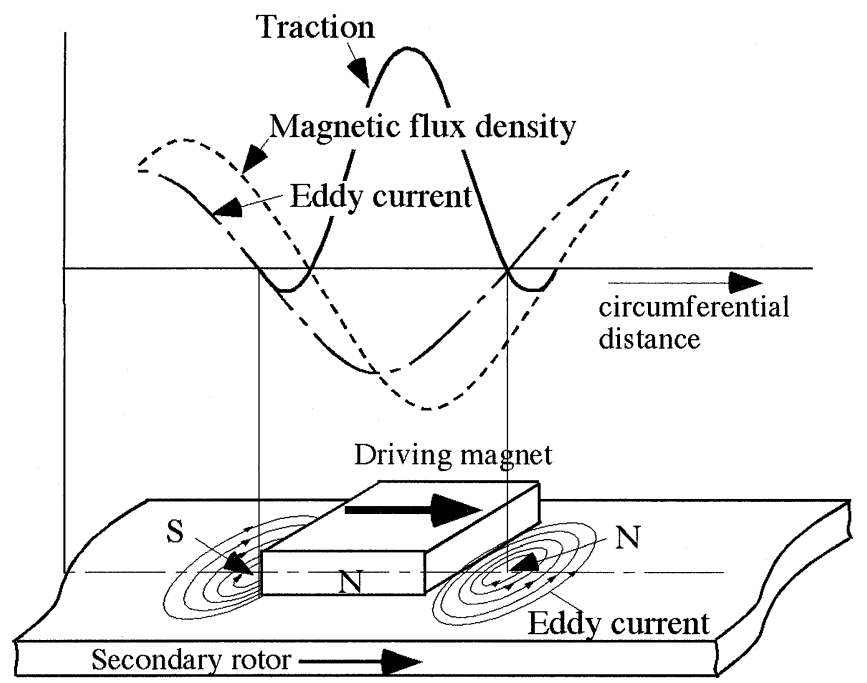

FIGURE 2

Driving principle of floating impeller. following equation in the circumferential direction is distributed.

$$
B=B_{0} \cos \left(\omega t-\frac{\pi x}{\tau}\right)
$$

The eddy current is generated on the rotor, when the secondary rotor exists in the magnetic field and a magnetic flux is generated. Traction force of rotational direction arises by the phase between magnetic field and the eddy current of the secondary rotor.

The variation of the traction force $F$ and normal force $F_{n}$ generated by the rotating magnetic field are estimated from the following equation by the linear motor theory (Ooi and White, 1974).

$$
\begin{gathered}
F=\frac{A \mu_{0}}{2} \cdot \frac{s R_{m} J_{s}^{2}}{(\sinh g \beta)^{2}+\left(s R_{m} \cosh g \beta\right)^{2}} \\
F_{n}=\frac{A \mu_{0}}{4} \cdot \frac{\left\{1-\left(s R_{m}\right)^{2}\right\} J_{s}^{2}}{(\sinh g \beta)^{2}+\left(s R_{m} \cosh g \beta\right)^{2}}
\end{gathered}
$$

From Equation (2), the traction force is large in the pump starting, and it becomes 0 at the synchronous speed. From Equation (3), the attraction force of normal direction produces, when the product of slip and magnetic Reynolds number $s R_{m}$ is smaller than 1.0. Moreover, the repulsion force will be produced, when $s R_{m}$ is bigger than 1 . From these results, the contact of the casing and secondary rotor is affected by change of radial force, as shown in Figure 3. In the case of secondary rotor made on nonmagnetic materials, the repulsion force of normal direction generates by effect of magnetic Reynolds number and slip. The secondary rotor of the magnetic substance becomes with the problem in order to realize the non contact rotation, because there is always in the attraction condition. Therefore, the secondary rotor was made on nonmagnetic material available of

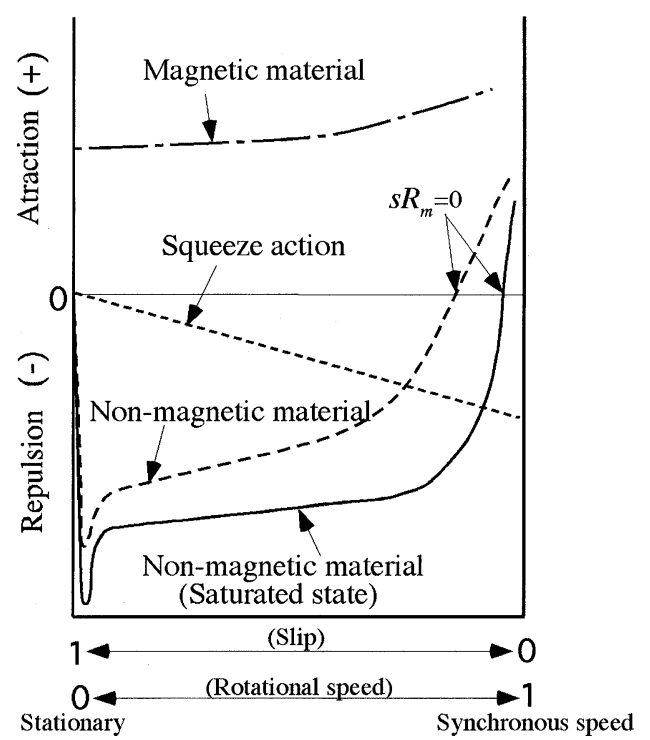

FIGURE 3

Relationship between normal force and slip. 


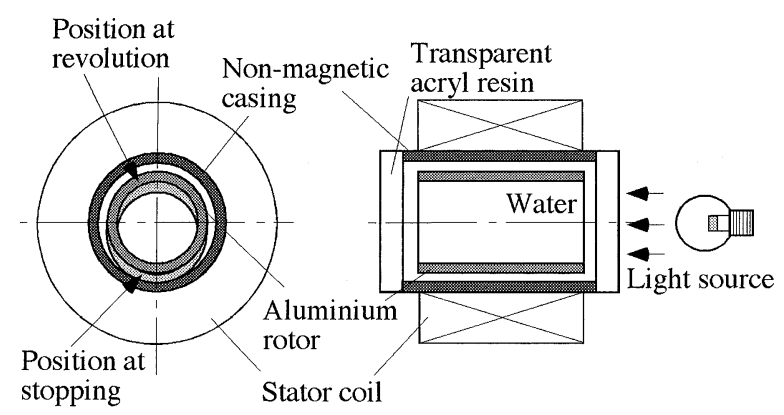

Sideveiw



FIGURE 4

Test device for visualization of floating rotor.

magnetic induction effect. In the case of synchronous speed of the secondary rotor and driving magnet, the radial force changes to attraction force. However, a squeeze effect arises due to liquid existence between partition and rotation rotor of the pump, and its effect prevents the contact between the rotational and stationary part.

For floating situation confirmation of the secondary rotor in the water, the visualization experiment was carried out by the equipment, as shown in Figure 4. The equipment was used for

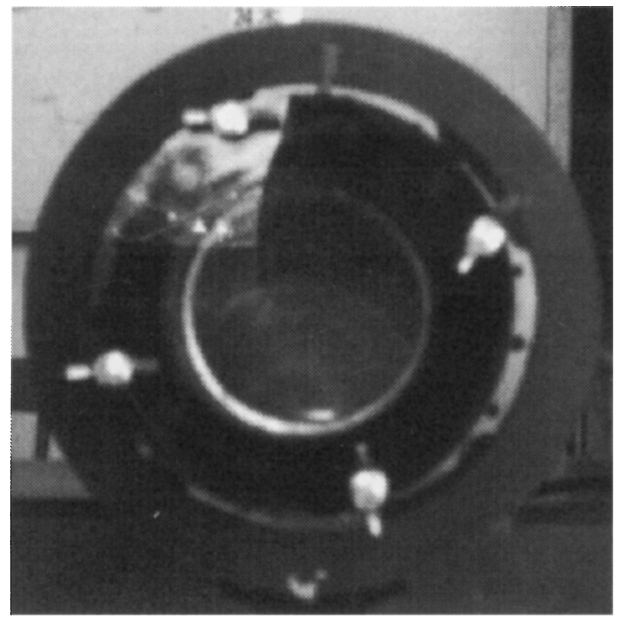

(a) Stopped state the sidewall in the transparent acrylic as a horizontal type. An aluminum cylinder of the non magnetic material was used for the secondary rotor, and the coil was utilized as a rotating magnetic field generator. Before the power charge to the coil on the stator occurred, the secondary rotor is touching at the casing bottom. The secondary rotor was rotating at the floating condition with the power charge. In the case of air in the casing, the rotor slides affect the attraction force approaching the synchronous speed. Because the liquid film effect does not work, the water is not located in the inside. The visualization results are shown in Figure 5. The floating situation was observed by the light source between casing bottom and rotor. From these experimental results, the tendency in Equations (2) and (3) was confirmed, and it was proven that the magnetic induction system was effective for the non contacting drive.

\section{CONSIDERATION OF THE FLOATING CENTIRFUGAL PUMP TYPE}

On the basis of the drive principle previously described, several kinds of pumps were examined as shown in Figure 6. Figure 6(a) shows a pump of the horizontal double suction type. The pump is the structure, which eliminates the axial thrust load in the faced impeller. However, a problem with the complexity of the structure, lower efficiency by the fluid friction resistance, and rotor deadweight appear. Figure 6(b) shows a horizontal single suction pump of the electromagnetic induction type with stator coil and secondary rotor made on magnetic substance. In this case, the impeller often touched the casing by the effect of rotor deadweight and axial thrust load. Figure 6(c) shows a single suction pump of the electromagnetically driven style with the vertical type. For the support of rotor weight, the magnet for the repulsion was installed, and the effect of

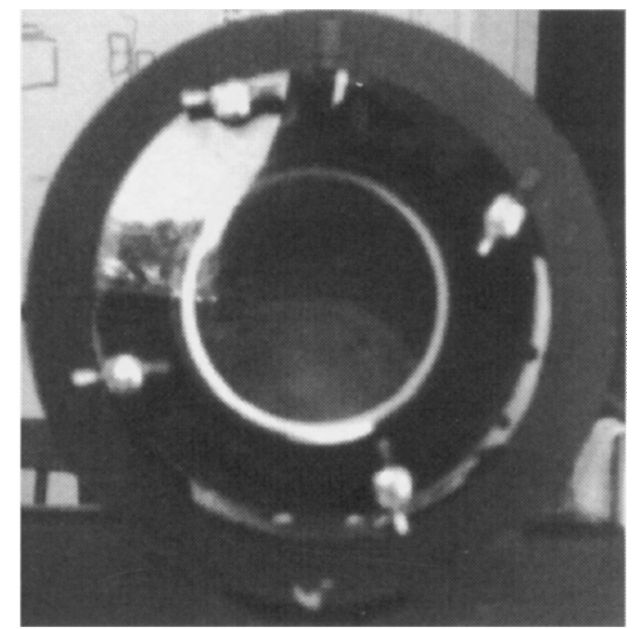

(b) Rotational state

FIGURE 5

Photograph of floating situation. 


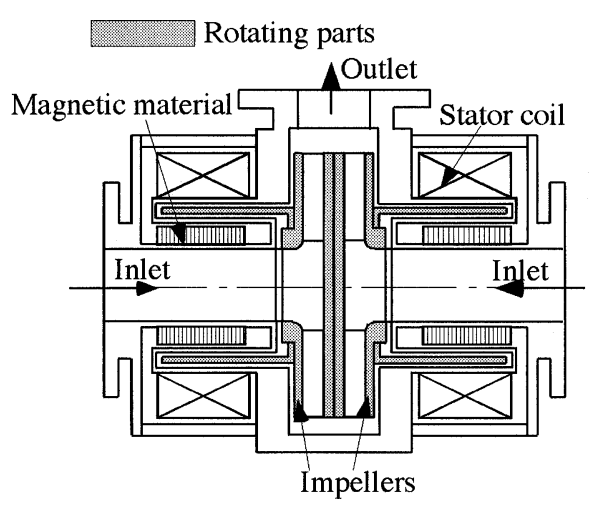

(a)

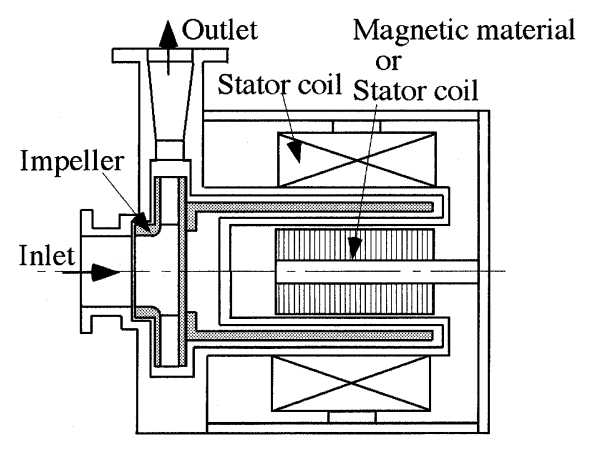

(b)

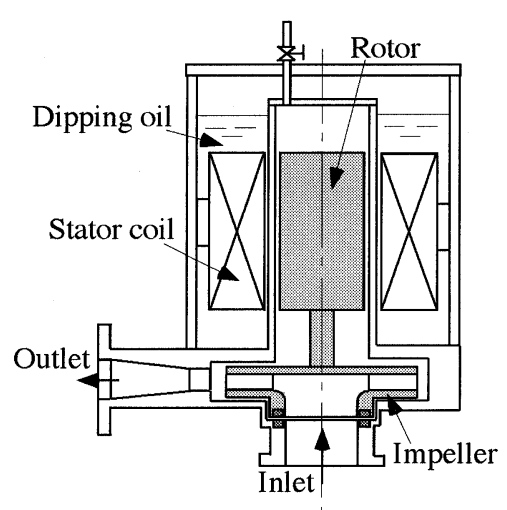

(c)

FIGURE 6

Consideration of the type of floating impeller pump.

(a) Horizontal double suction type (electromagnetic drive),

(b) Horizontal single suction type (electromagnetic drive),

(c) Vertical single suction type (electromagnetic drive).

the temperature rise of the coil and transportation fluid was confirmed.

From the above progress of development, the pump of magnet inductive type used by the motor as a rotating magnetic field generator was developed. The developed floating centrifugal pump of the magnet induction drive is shown in Figure 7. As a result,

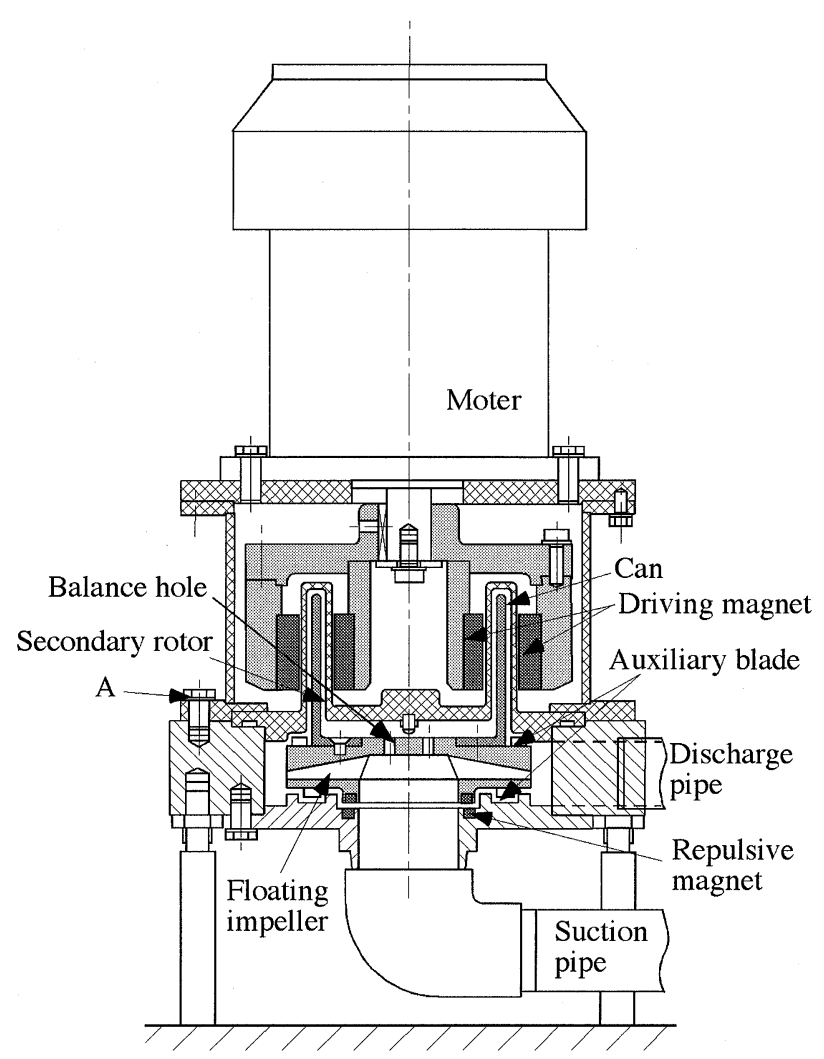

(a) Assembly drawing

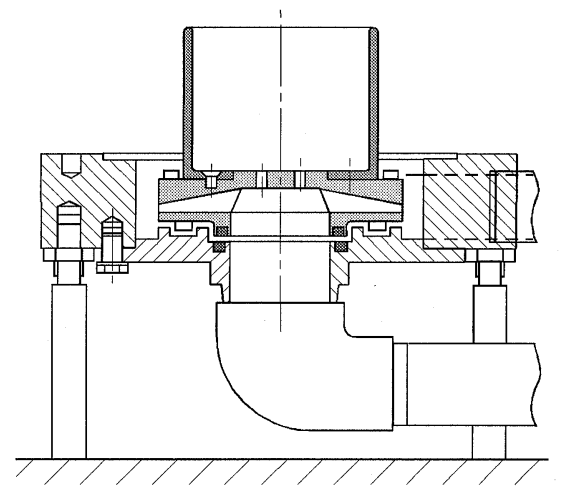

(b) Decomposed state

\section{FIGURE 7}

The structure of the test pump.

it was clarified that the rotating magnetic field generator by the magnet was more advantageous than the electromagnetic drive as outlined below.

(1) The rotating magnetic field generator by use of the magnet is no loss of the magnetism generator. The influence of magnetic gap and partition thickness is small.

(2) The rotational speed of the impeller is dependent on the motor rotational speed regardless of the magnetic pole number. Furthermore, the torque can be adjusted in the magnet pole number. 
TABLE 1

Specifications of Test Pump

\begin{tabular}{lcc}
\hline Suction diameter & $\mathrm{D}_{\mathrm{s}}$ & $50 \mathrm{~mm}$ \\
Discharge diameter & $\mathrm{D}_{\mathrm{d}}$ & $40 \mathrm{~mm}$ \\
Impeller: & & \\
$\quad$ Outlet radius & $\mathrm{R}_{2}$ & $125 \mathrm{~mm}$ \\
Outilet width & $\mathrm{b}_{2}$ & $5 \mathrm{~mm}$ \\
$\quad$ Number of vanes & $\mathrm{Z}_{\mathrm{i}}$ & 6 \\
Rating: & & \\
$\quad$ Rotational speed & $\mathrm{N}$ & $3460 \mathrm{rpm}$. \\
Flow rate & $\mathrm{Q}$ & $0.25 \mathrm{~m}^{3} / \mathrm{min}$ \\
Total head rise & $\mathrm{H}$ & $21 \mathrm{~m}$ \\
\hline
\end{tabular}

(3) The temperature rise dose not show in the magnetic driving system.

(4) In comparison with the magnet pump, the magnetic induction system is no step out of the secondary rotor, and it is possible to raise the rotational speed smoothly.

\section{THE STRUCTURE OF THE DEVELOPED PUMP}

Figure 7(a) shows the structure of the developed floating centrifugal pump, and Table 1 shows the design specification of the developed pump. The specific speed of the test pump is 176 $\left(\mathrm{m}^{3} / \mathrm{min}, \mathrm{m}, \mathrm{rpm}\right)$. The maintenance of a pump becomes easiest by adopting the magnet induction type. Furthermore, the developed pump can be simply decomposed by detaching clamping bolt A as shown in Figure 7(b). For the lightweight of the rotor

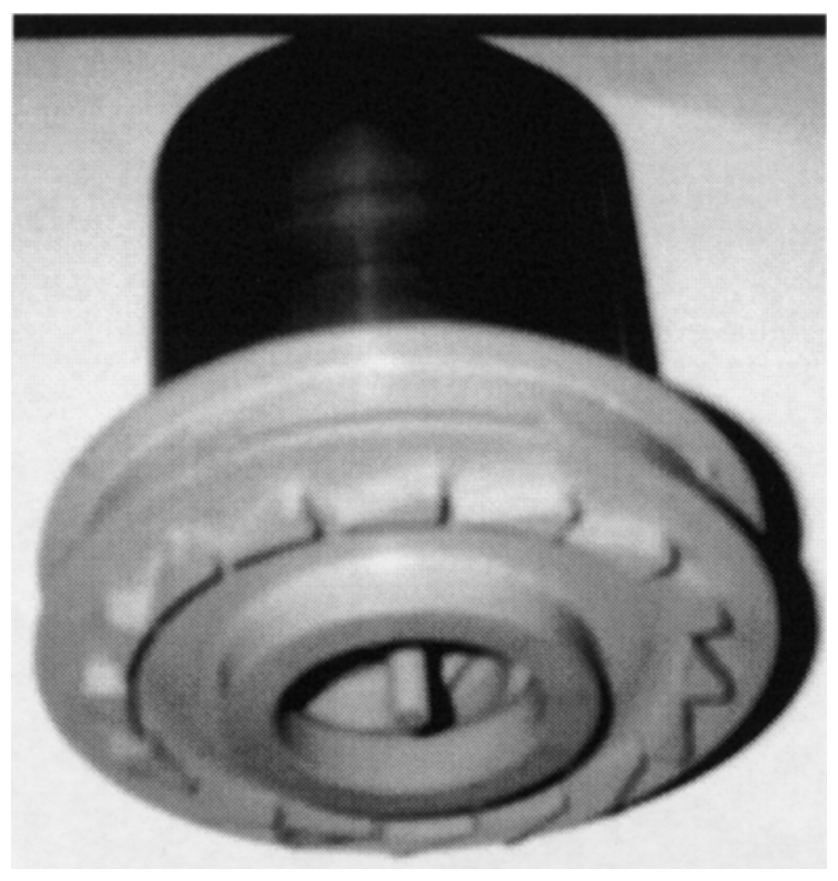

FIGURE 8

Photograph of the test impeller.

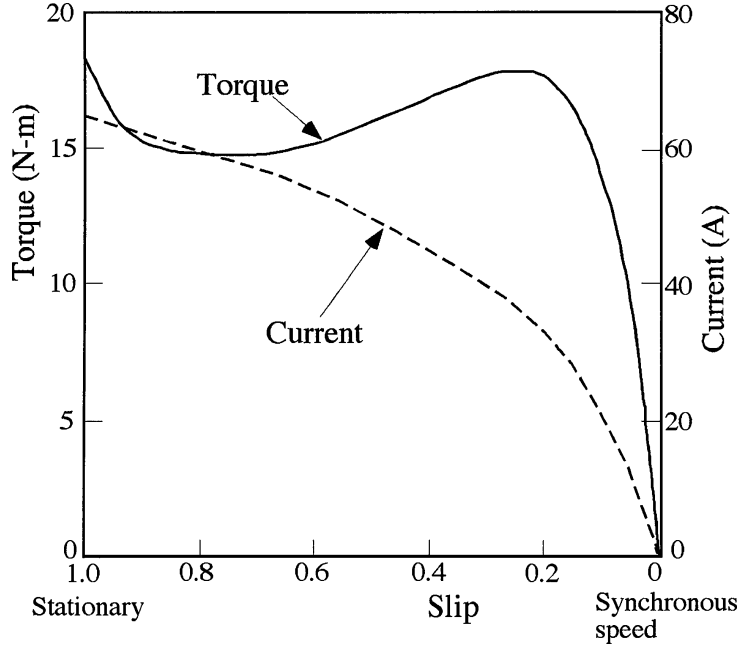

FIGURE 9

Motor current and driving torque by the slip.

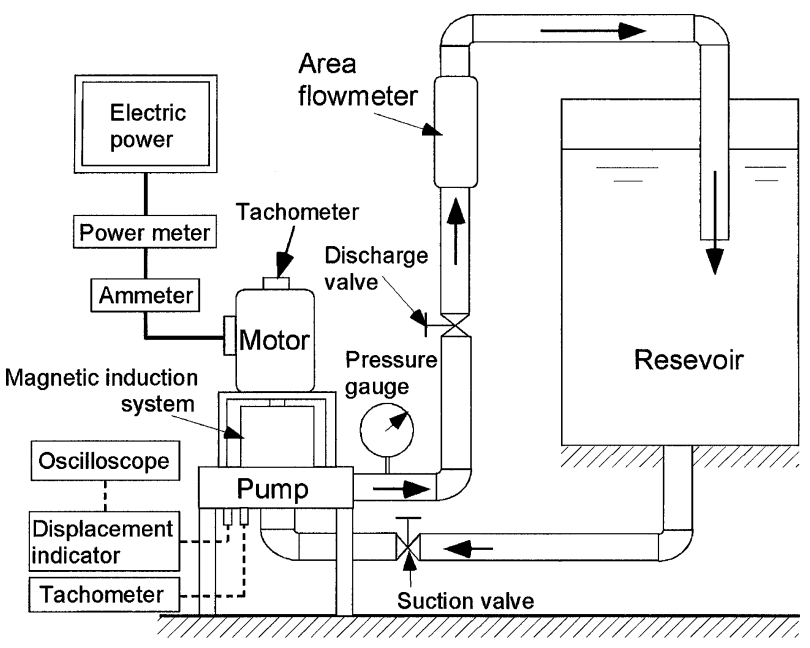

FIGURE 10

Performance test equipment of pump.

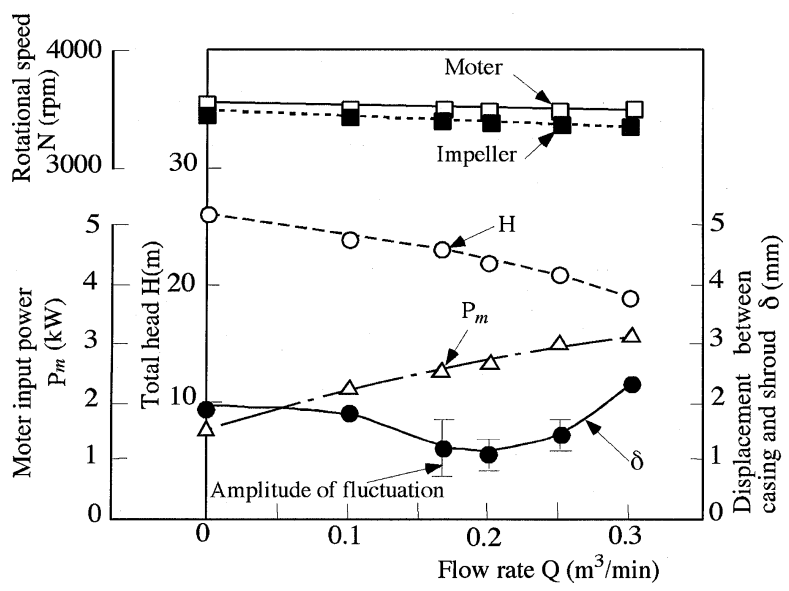

FIGURE 11

Performance curve in driving at $60 \mathrm{~Hz}$ and floating distance of the impeller. 


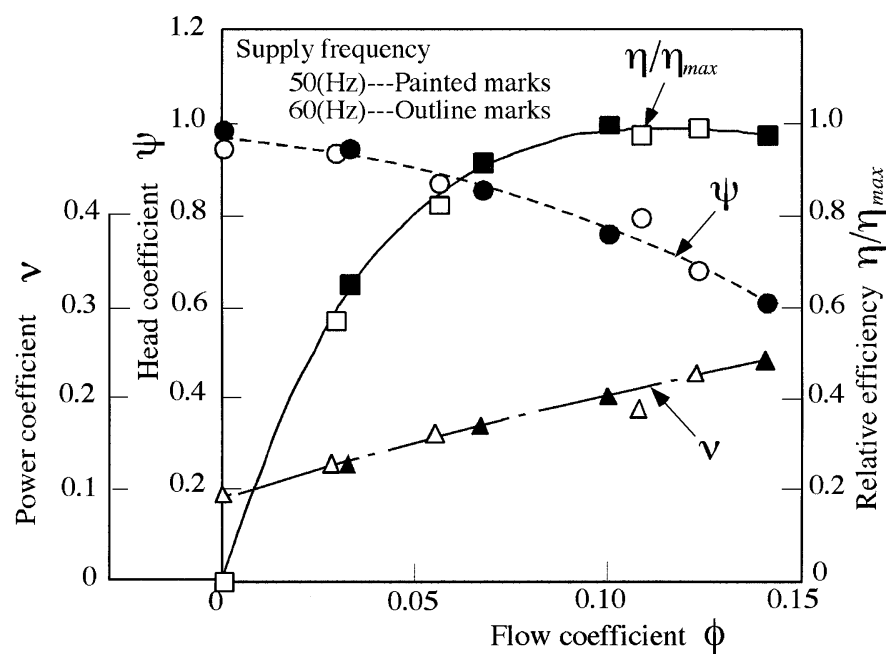

FIGURE 12

Characteristic curve of the test pump.

including the impeller, the impeller is manufactured in the resin. In addition to the effect of repulsive force, the secondary rotor is manufactured in light aluminum of the non magnetic material. The surface of the secondary aluminum rotor is coated in gold plating and resin in order to prevent metal ion elution. Considering the touch by the radial vibration of the rotor, the adequate clearance was set between rotor and casing. The balance hole was installed in order to reduce the axial thrust by the pressure difference between the shroud and disk side of the impeller. In addition to the balance hole, the aileron blade was installed in the shroud and disk side in order to utilize a liquid film effect and adjustment of pressure. A photograph of the developed floating impeller is shown in Figure 8. Figure 9 shows steady-state characteristics of the trial pump magnetic drive system. The figure shows motor current and driving torque with the slip. ( $\mathrm{s}=0$ is a stopping state; $s=1$ is in case of the synchronous speed.) The torque is big from the stopping state of the secondary rotor to near $\mathrm{s}=0.2$, and it lowers afterwards. The tendency agrees with the linear motor.

\section{TEST METHOD AND PUMP PERFORMANCE}

Figure 10 shows the performance test equipment of a floating centrifugal pump. The water from the upper tank returns to the tank through the discharge pipe, valve, and flowmeter. The flow rate was obtained by an area flowmeter, and the total head are measured from the delivery pressure as a base of upper tank. Furthermore, the rotational speed of the drive magnet detects by the tachometer installed in the motor and impeller rotational speed measured by photodetector. The floating distance between the impeller and casing is measured by a displacement sensor of the eddy type with a high frequency response installed in the casing wall surface. The time-dependent signal of the distance is confirmed by the synchroscope. Furthermore, the supplied power to the motor is required using

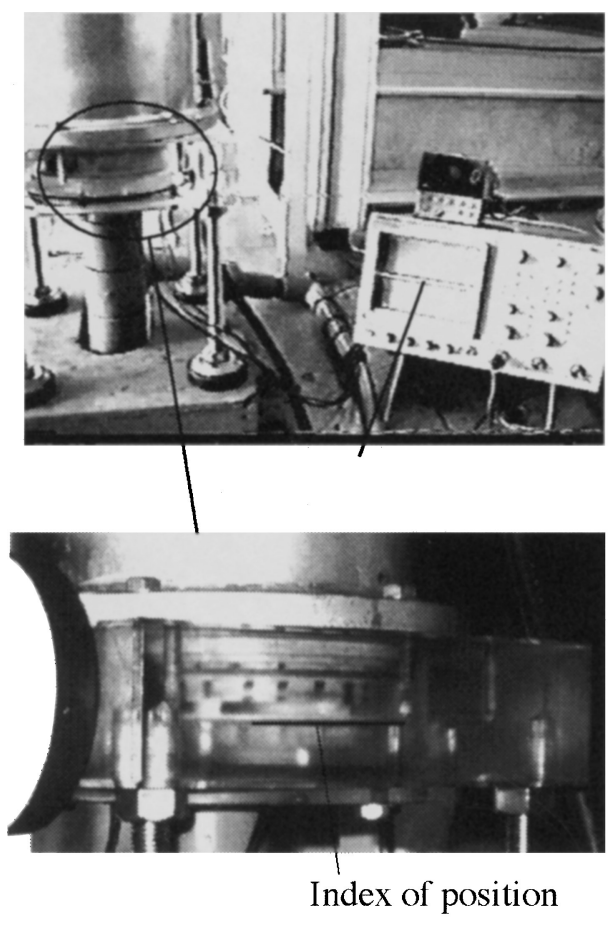

(a) Stopped state

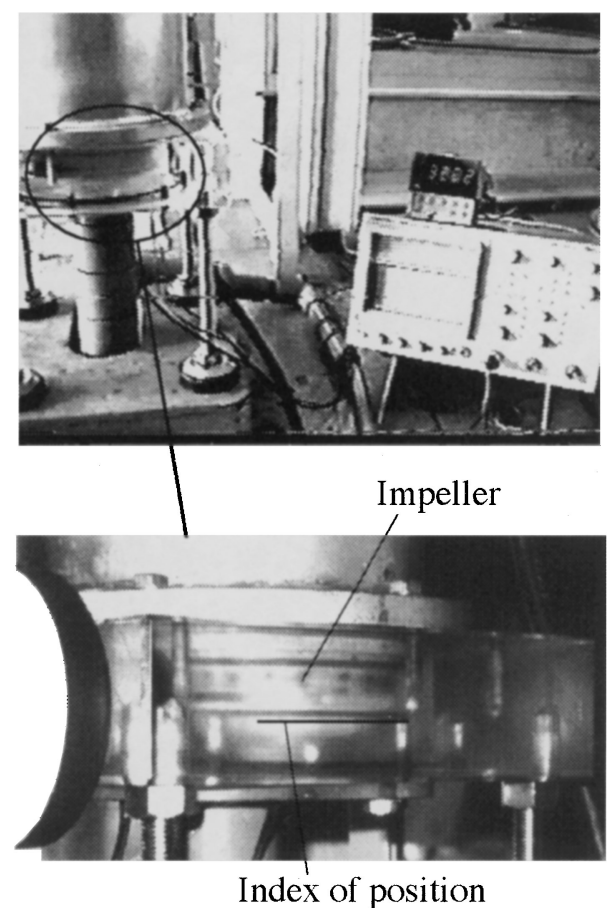

(b) Rotational state

FIGURE 13

Picture of floating state of the test impeller. 
wattmeter, ammeter, and voltmeter. The supplied power frequency to motor is adjusted by a frequency converter at $50 \mathrm{~Hz}$ and $60 \mathrm{~Hz}$.

Figure 11 shows a performance curve of a developed pump in driving at $60 \mathrm{~Hz}$ and a floating distance of the impeller. The total head of a test pump decreases with the increasing in flow rate similar to the performance of a general centrifugal pump. The supplied power to motor increases with the flow rate, and the difference of impeller and motor rotational speed increases. The floating distance is the least near the design point, and it increases by an increase and decrease of flow rate. Moreover, the fluctuation of floating distance is small in the design point, and it increases in the small flow rate area due to pressure fluctuation and internal flow.

Figure 12 shows characteristic curves in the case of $50 \mathrm{~Hz}$ and $60 \mathrm{~Hz}$ at supply power frequency of motor. The variation of power coefficient $\nu$, head coefficient $\psi$, and efficiency $\eta$ with flow coefficient $\phi$ are indicated. In this result, the characteristic curve shows good agreement on two cases, because there was no mechanical contact part. The maximum efficiency point appeared near the design condition, and the high-efficient region spread comparing with a general centrifugal pump. After the performance test, the floating state of the test impeller was investigated to observe from the outside of a transparent casing.

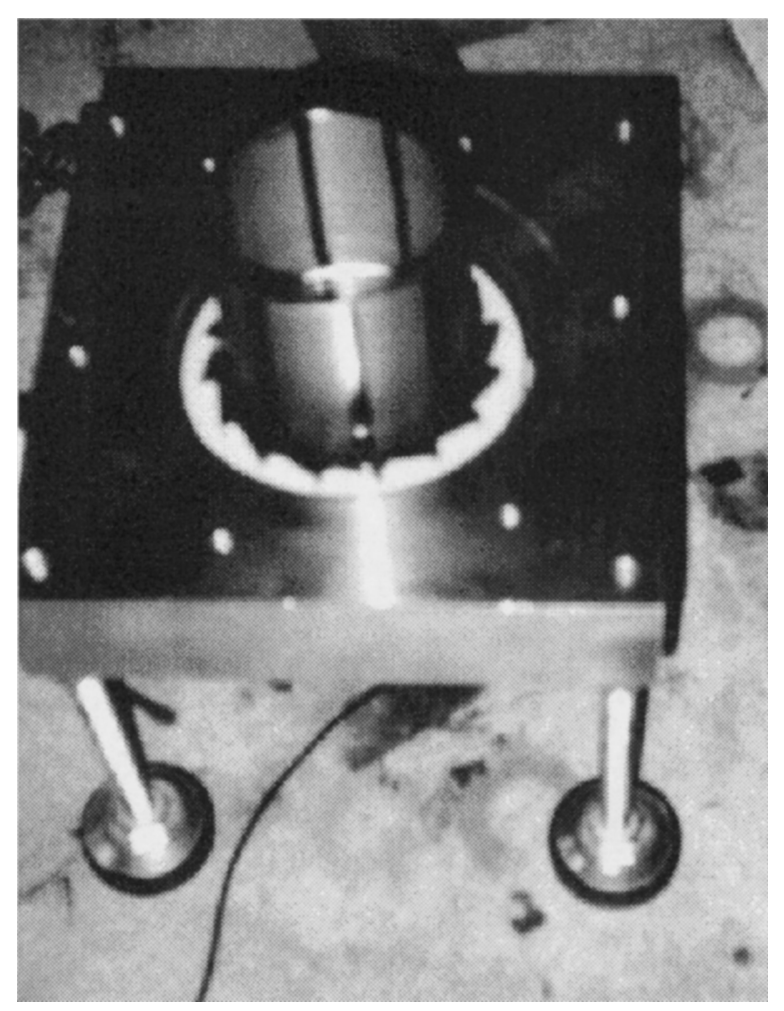

FIGURE 14

Contact confirmation photograph of the secondary rotor.
Figure 13 show the picture of the floating state of impeller. The shroud side of the impeller is touching at the casing's lower side before the rotation, and it is levitating the rotational condition as shown in Figure 13(b). The floating state of perpendicular direction was confirmed from the output signal of the displacement sensor and a picture from a video camera. However, the contact to radial direction between secondary the rotor and partition is not confirmed by the observation from casing outside due to the existence of a non contact driving mechanism. Furthermore, the displacement sensor cannot utilize the present experiment to receive the effect of the magnetic field. Therefore, the oil painting method was applied, and the non contacting condition of dead angle was confirmed in the observation of the peeling of the paint after the operation. An example of the observation results is shown in Figure 14. In the wide operating range, non contact condition of test impeller was confirmed by the above experimental result.

\section{CONCLUSIONS}

As a result of development, operation, and performance test of non contacting magnetic-drive style pump with the floating impeller, the following was made clear. The installation area of the pump by the vertical type non contacting magnetic drive system was decreased. Using the magnet induction method, decomposition and assembly of the test pump became easy. In effect of the aileron blade and balance hole, the reduction of the axial thrust was possible, and the floating impeller was realized. The characteristic similarity of the developed floating centrifugal pump was confirmed.

In the future, it is necessary that the experimental research on stability of the floating impeller in low flow rate and transient condition carry out measurement of instantaneous displacement of rotating impeller and pressure field.

\section{NOMENCLATURE}

$A$ area

$B$ magnetic flux density

$F$ traction force

$F_{n} \quad$ normal force

$f$ frequency

$g$ gap

$J_{s} \quad$ surface current

$R_{m}$ magnetic Reynolds number $\left(=V_{s} \mu_{0} / \rho_{r}\right)$

$s \quad \operatorname{slip}\left(=1-V_{2} / V_{s}\right)$

$V_{2}$ secondary rotor speed

$V_{s}$ synchronous speed

$\beta \quad$ wavelength ratio

$\eta \quad$ efficiency

$\mu_{0} \quad$ permittivity

$v$ power coefficient

$\rho_{r} \quad$ surface resistance

$\tau \quad$ distance between magnetic pole

$\phi \quad$ flow coefficient 
$\psi \quad$ head coefficient

$\omega$ turning angular velocity of the magnetic field $(=2 \pi f)$

\section{REFERENCES}

Akamatsu, T. 1994. Centrifugal blood pump. J. Turbomachinery 22(1):40-46 (in Japanese).
Ooi, B. T., and White, D. C. 1974. Traction and normal forces in the linear induction motor. IEEE Trans. Power Apparatus and System PAS-89(4):638-645.

Pfleiderer, C. 1961. Die Kreiselpumpen. Berlin: Springer Verlag.

Stepanoff, A. J. 1948. Centrifugal and axial flow pumps. New York: John Wiley \& Sons. 

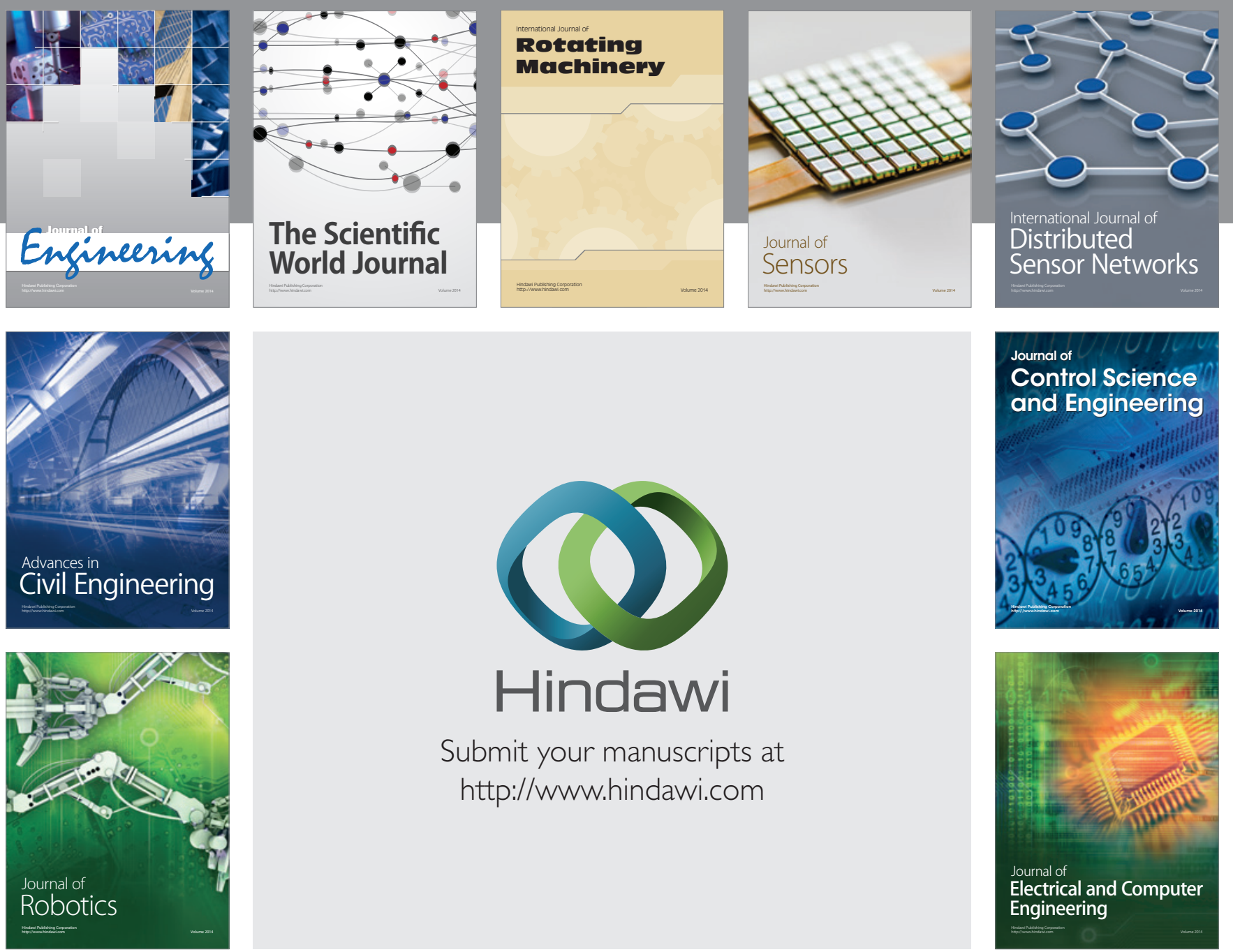

Submit your manuscripts at

http://www.hindawi.com
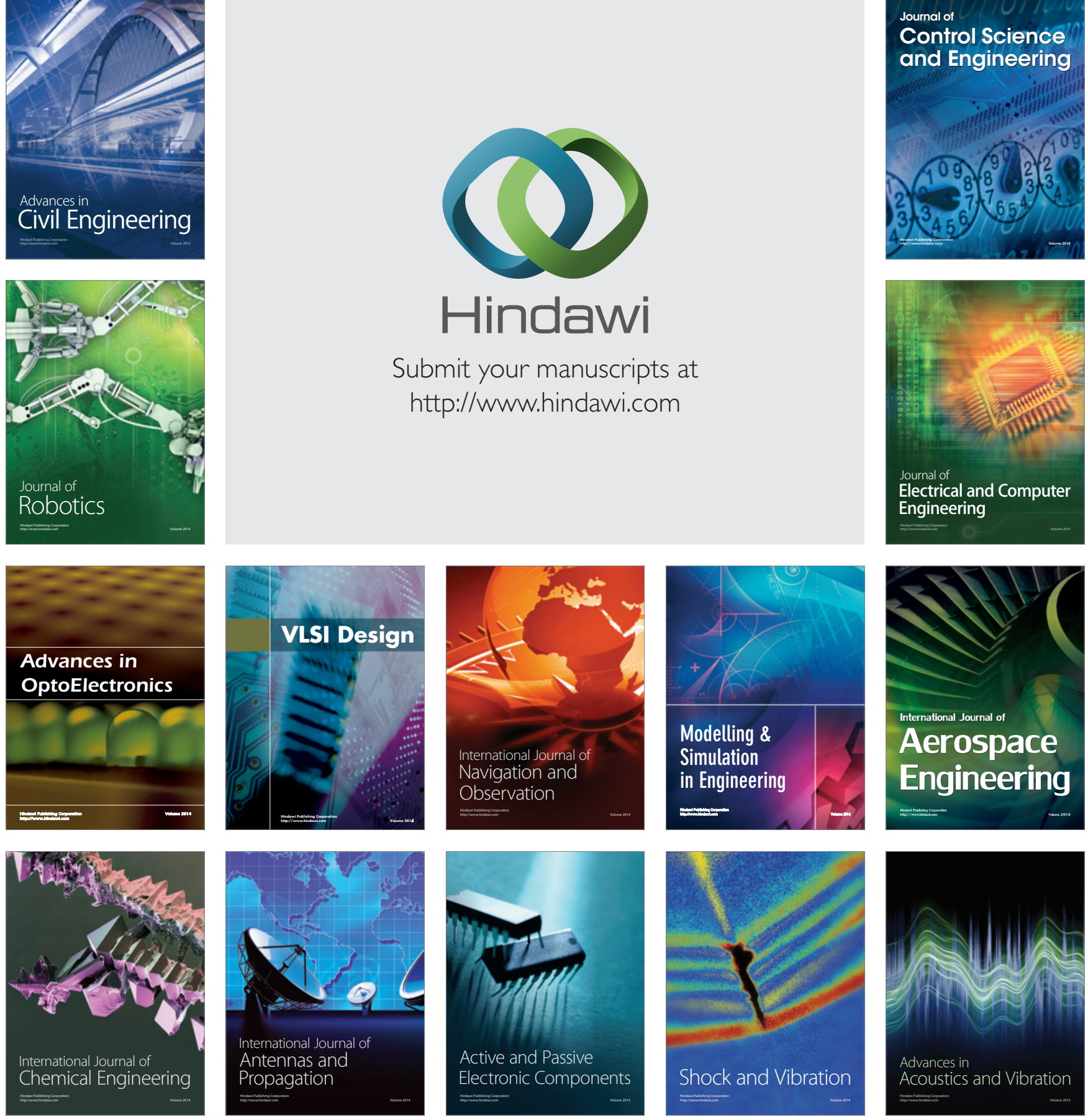